\title{
Stroke and Thromboembolism in Patients with Heart Failure and Sinus Rhythm: A Matter of Risk Stratification?
}

\author{
Angelica Rivas ${ }^{1}$ Mandy N. Lauw ${ }^{2}$ Renate B. Schnabel ${ }^{3,4}$ Mark Crowther ${ }^{1}$ Harriette G. C. Van Spall ${ }^{1,5,6,7}$
}

${ }^{1}$ Department of Medicine, McMaster University, Hamilton, Ontario, Canada

2 Department of Hematology, Erasmus University Medical Center, Rotterdam, The Netherlands

${ }^{3}$ Department of Cardiology, University Heart and Vascular Centre UKE Hamburg, Hamburg, Germany

${ }^{4}$ German Center for Cardiovascular Research (DZHK), partner site Hamburg, Germany

5 Population Health Research Institute, McMaster University, Hamilton, Ontario, Canada

${ }^{6}$ Department of Health Research Methods, Evidence, and Impact, McMaster University, Hamilton, Ontario, Canada

7 Research Institute of St. Joseph's, Hamilton, Ontario, Canada
Address for correspondence Harriette G. C. Van Spall, MD, MPH, McMaster University, 20 Copeland Avenue, David Braley Research Building, Suite C3-117, Hamilton, Ontario L8L 0A3, Canada (e-mail: harriette.vanspall@phri.ca).

Thromb Haemost 2022;122:871-878.

\begin{abstract}
Patients with heart failure (HF) in sinus rhythm (SR) experience an increased incidence of thromboembolic events including stroke. Among patients with HF, high-quality evidence supports the use of oral anticoagulation when atrial fibrillation is present, but the benefit of anticoagulation in SR in the absence of other known indications for anticoagulation is unclear. In four randomized controlled trials (RCTs), warfarin did not improve a composite of clinical outcomes compared with aspirin or placebo in patients with HF with reduced ejection fraction (HFrEF) and SR. A recent RCT assessed the efficacy of the direct oral anticoagulant rivaroxaban versus placebo in patients with HFrEF (including mildly reduced ejection fraction), SR, and coronary artery disease. While rivaroxaban had a neutral effect on the primary composite outcome of myocardial infarction, stroke, or all-cause mortality, exploratory analyses revealed a significant reduction in strokes. It is thus possible that a subgroup of patients with HFrEF who are at high risk of stroke may benefit from anticoagulation. The challenge is

Keywords

- anticoagulants

- heart failure

- ischemic stroke

- sinus rhythm

- thromboembolism to adequately identify this subgroup and to balance the potential benefit of anticoagulation with the risk of major bleeding. There is also an unmet need for evidence around anticoagulation in HF with preserved ejection fraction and SR. This review explores the current evidence around anticoagulation in patients with HF and SR, identifies challenges regarding outcome definitions and patient selection, and offers suggestions for future research.
\end{abstract}

received

October 15, 2021

accepted after revision

January 18, 2022

published online

January 19, 2022 (c) 2022. Thieme. All rights reserved. Georg Thieme Verlag KG,

Rüdigerstraße 14,

70469 Stuttgart, Germany
DOI https://doi.org/

10.1055/a-1745-2083.

ISSN 0340-6245. 


\section{Introduction}

Patients with heart failure (HF) are at an increased risk of morbidity and mortality. Advances in medical management including guideline-directed medical therapy and implantable cardioverter-defibrillators have improved prognosis. Nevertheless, stroke continues to be a devastating complication in patients with HF. ${ }^{1,2}$ Among patients with HF, it is hypothesized that the combination of endothelial dysfunction, comorbid conditions increasing the hypercoagulability of blood, and stasis of flow from atrial and ventricular dysfunction produces a prothrombotic state and subsequently increases the risk of intracardiac thrombi and stroke $^{1,3}$ (- Fig. 1). The estimated incidence of stroke among patients with HF in sinus rhythm (SR) is 1.1 to 1.6 per 100 patient-years. ${ }^{4,5}$ Ischemic stroke is the presenting event in $82 \%$ of patients, with the majority of these first stroke events being fatal or causing significant disability. ${ }^{5}$ This represents a 1.5- to 2.1-fold risk of ischemic stroke relative to patients in SR without HF. ${ }^{6}$ However, current guidelines recommend anticoagulation in HF only in those patients with atrial fibrillation (AF) or another indication for anticoagulation, such as a prior history of thromboembolism or a prosthetic valve, with no specific recommendations based on ejection fraction. The purpose of this review is to summarize the literature around recommendations for anticoagulation in patients with HF and SR, and to identify challenges and opportunities regarding outcome definitions and patient selection in light of emerging evidence.

\section{Clinical Trial Evidence: Anticoagulation in Heart Failure and Sinus Rhythm}

The benefit of anticoagulation in reducing the risk of stroke has been well established in patients with HF and concomitant $\mathrm{AF}$, but evidence to support the use of anticoagulation in patients with HF and SR is lacking. AF is a well-defined

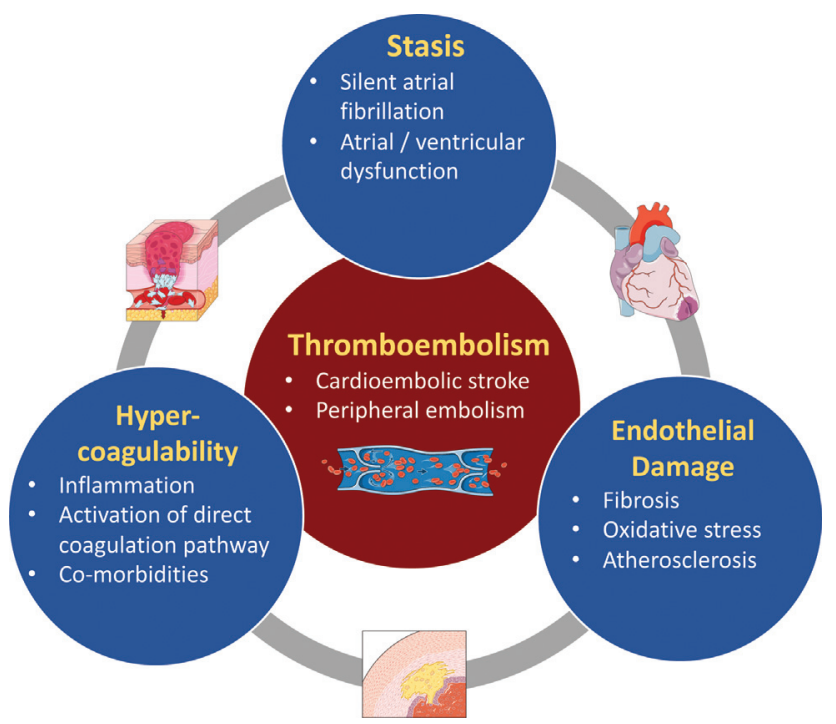

Fig. 1 Thrombosis formation and specific heart failure-related components that pertain to Virchow's triad. independent risk factor for ischemic stroke. Earlier cohort studies with 30 years of follow-up in the general population revealed that $12 \%$ of strokes were associated with $\mathrm{AF}^{7}$ More recent evidence has suggested that $A F$ is present in $51 \%$ of patients with HF and stroke, ${ }^{7}$ and that patients with both comorbidities have a stroke incidence rate of $1.6 \%$ per year. $^{4}$ These findings suggest that AF may be a strong predictor of future stroke risk in patients with HF. AF is common in HF, estimated at $50 \%$ and even more common in patients with $\mathrm{HF}$ with preserved ejection fraction (HFpEF) who make up approximately half of the population of patients diagnosed with $\mathrm{HF}^{8}$ Both conditions are associated with similar risk factors including older age, diastolic dysfunction, and agerelated comorbidities including hypertension, obesity, and sleep apnea. ${ }^{9}$ Relative to HF with reduced ejection fraction (HFrEF), HFpEF may be associated with greater inflammation and oxidative stress and a higher risk of (AF-related) ischemic stroke. ${ }^{10}$ Indeed, in an age- and sex- matched registry study of Korean patients with AF, the annual incidence of stroke and systemic embolism in patients with HFpEF and HFrEF was 2.8 and $1.1 \%$, respectively. ${ }^{11}$ Notably, the severity of $\mathrm{HF}$ symptoms may be associated with AF prevalence, with one study reporting less than $5 \% \mathrm{AF}$ in patients with mild $\mathrm{HF}$ (NHYA [New York Heart Association] class I) and 50\% in patients with severe HF (NHYA class IV). ${ }^{8}$

There have been five randomized controlled trials in over 9,000 patients with HFrEF and SR to assess the efficacy and safety of anticoagulant therapies (warfarin vs. placebo or antiplatelet agents; rivaroxaban vs. placebo) ${ }^{12-14}$ (- Fig. 2 and - Table 1). Methodological limitations in and knowledge gaps unaddressed by these trials preclude definitive conclusions.

The Warfarin/Aspirin Study in Heart Failure (WASH) open-label trial randomized 279 patients with HF, SR, and left ventricular ejection fraction (LVEF) $\leq 35 \%$ to warfarin, aspirin, or no antithrombotic therapy. After a mean followup of 27 months, neither warfarin nor aspirin improved the primary composite outcome of death, nonfatal myocardial infarction (MI), or nonfatal stroke relative to no antithrombotic therapy ${ }^{12-14}$ (-Table 1). The Heart Failure Long-Term Antithrombotic Study (HELAS) enrolled 197 patients with HF, LVEF $<35 \%$, and either ischemic heart disease (history of MI) or dilated cardiomyopathy. The trial assessed the efficacy of warfarin versus aspirin in the ischemic heart disease cohort, and warfarin versus placebo in the dilated cardiomyopathy cohort. There were no significant differences between groups in the primary composite outcome of nonfatal stroke, peripheral or pulmonary embolism, recurrent MI, rehospitalization, exacerbation of HF, or all-cause death $^{12-14}$ (- Table 1). Importantly, both trials were small and had inadequate statistical power to detect betweengroup differences. Hence, inferences from these trials cannot be reliably made.

The Warfarin and Antiplatelet Therapy in Chronic Heart Failure (WATCH) double-blinded trial enrolled 1,587 patients with HF (NYHA class II-IV, LVEF $\leq 35 \%$ ) and SR who had been treated with a diuretic and angiotensinconverting enzyme inhibitor for 60 days. The trial found no 


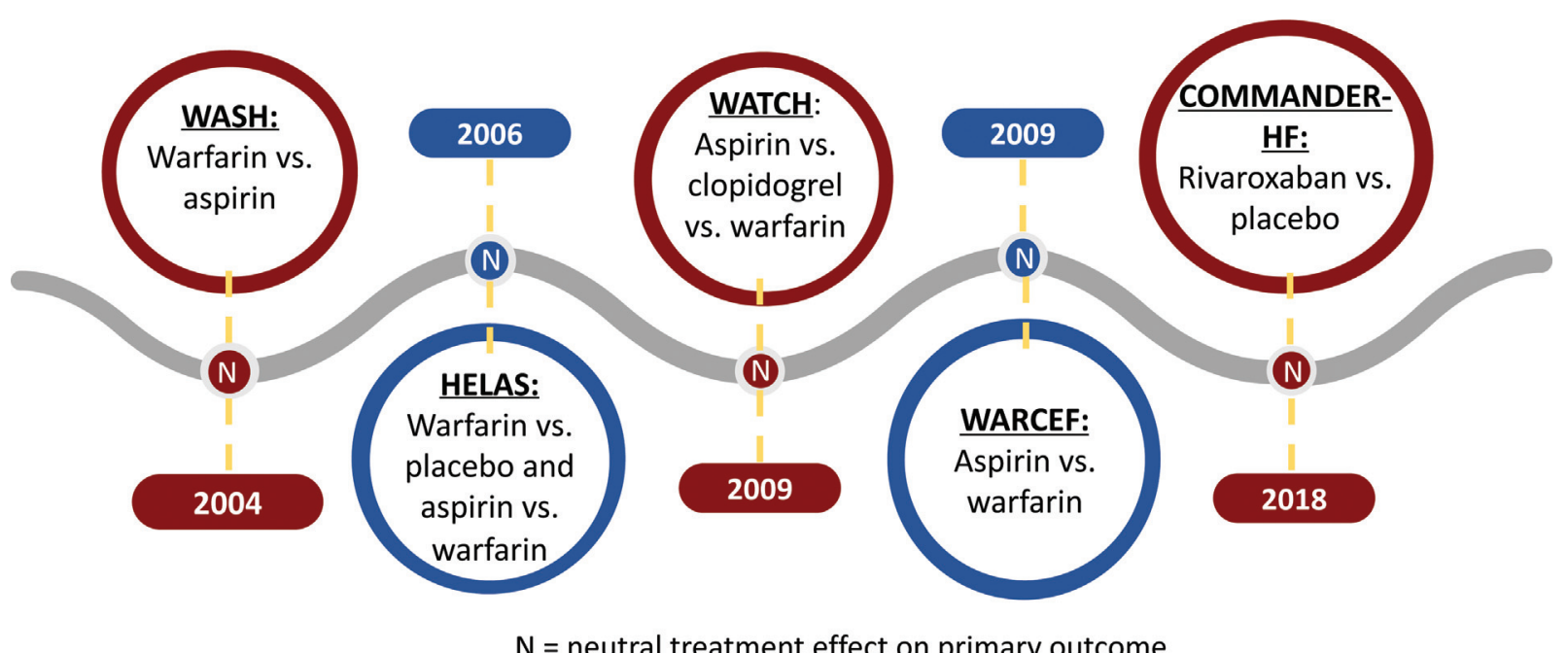

Fig. 2 Timeline of landmark randomized clinical trials assessing anticoagulation in patients with heart failure in sinus rhythm.

significant treatment benefit of warfarin versus aspirin and warfarin versus clopidogrel on the primary composite outcome of time to first death, nonfatal MI, or nonfatal stroke after a median follow-up of 21 months. ${ }^{12-14}$ However, warfarin was associated with fewer nonfatal strokes compared with aspirin or clopidogrel (-Table $\mathbf{1})$. This finding was consistent with the Warfarin versus Aspirin in Reduced Cardiac Ejection Fraction (WARCEF) trial that randomized 2,305 patients with chronic HF (NHYA class I-IV, LVEF $\leq 35 \%$ ) and SR to warfarin versus aspirin. After a mean follow-up of 42 months, warfarin did not improve the primary composite outcome of time to first ischemic stroke, intracerebral hemorrhage, or death from any cause ${ }^{12-14}$ (-Table 1 ). While warfarin reduced the rate of ischemic stroke relative to aspirin, this clinical benefit was associated with a twofold increased risk of major bleeding.

With the arrival of the direct oral anticoagulants, COMMANDER-HF sought to re-evaluate the possible benefits of anticoagulation therapy in HF. This event-driven trial randomized 5,022 patients with $\mathrm{HF}, \mathrm{LVEF} \leq 45 \%$, and coronary artery disease to low-dose rivaroxaban versus placebo. After a median follow-up of 21 months, rivaroxaban did not significantly reduce the primary composite outcome of all-cause mortality, MI, or stroke ${ }^{15}$ (- Table 1). However, in exploratory analyses, rivaroxaban was associated with a reduction in thromboembolism, when sudden/unwitnessed deaths were excluded from the thromboembolic composite outcome (MI, ischemic stroke, symptomatic pulmonary embolism, or systematic pulmonary embolism). ${ }^{16}$ Further exploratory analysis of COMMANDER-HF suggested that low-dose rivaroxaban may reduce time to first event of any stroke or transient ischemic attack by $32 \%{ }^{5}$ There was no statistically significant difference in fatal bleeding or bleeding into a critical space with the possibility for permanent disability. ${ }^{5}$

Whereas some of the trials are not adequately designed to reliably make inferences, the breadth of evidence suggests that anticoagulation may prevent thromboembolic events among patients with HFrEF and SR, but this benefit may be offset by bleeding. Two recent meta-analyses of WASH,
HELAS, WATCH, WARCEF, and COMMANDER-HF showed that anticoagulation consistently reduced stroke risk. ${ }^{12,13}$ However, there was a significant increase in bleeding, a consistent finding across three of the largest trials regardless of the type of oral anticoagulant. Furthermore, the inclusion of all-cause death in the primary composite outcomes may attenuate the estimated benefit because stroke or bleeding events in this population represent a small proportion of deaths (2.5 and $0.4 \%$, respectively) relative to other causessuch as pump failure or arrhythmias-that are not responsive to anticoagulation. ${ }^{15}$ Future trials will need to consider the inclusion of cardiovascular death and all-cause death as secondary outcomes. Finally, the effect of anticoagulation in HFpEF and SR remains to be investigated.

\section{Can Risk Stratification Identify Patients Who May Benefit from Anticoagulation?}

\section{Stroke and Bleeding: Clinical Risk Prediction}

Risk stratification may identify a subset of patients with HF and SR that could benefit from anticoagulation. The challenge is to ensure that the thromboembolic risk outweighs the bleeding risk to warrant benefit from antithrombotic therapy (-Fig. 3). Risk factors associated with an increased risk of thromboembolism in patients with $\mathrm{HF}$ and SR include: older age, a history of stroke, diabetes, a higher body mass index, worsening NHYA class (III/IV), N-terminal pro B-type natriuretic peptide (NT-proBNP), and cardiomegaly. ${ }^{4,17}$ In patients with HF in SR (NHYA class II-IV, LVEF $\leq 40 \%$ ), a previous history of stroke, in particular, is a strong predictor for recurrent stroke (hazard ratio: $1.81,95 \%$ confidence interval: $1.19-3.74, p<0.01){ }^{4}$ Following an ischemic stroke, older age ( $\geq 75$ years), HF and high levels of NT-proBNP $(>400 \mathrm{pg} / \mathrm{mL})$, atrial tachyarrhythmias, and left atrial enlargement are associated with subsequent detection of $\mathrm{AF}^{18}$ Type of stroke (arterio-arterial embolism, cryptogenic or embolic stroke of undetermined source, and cardiac sources of stroke) is also associated with subsequent AF detection. ${ }^{18}$ Together, these predictors may be helpful for determining 


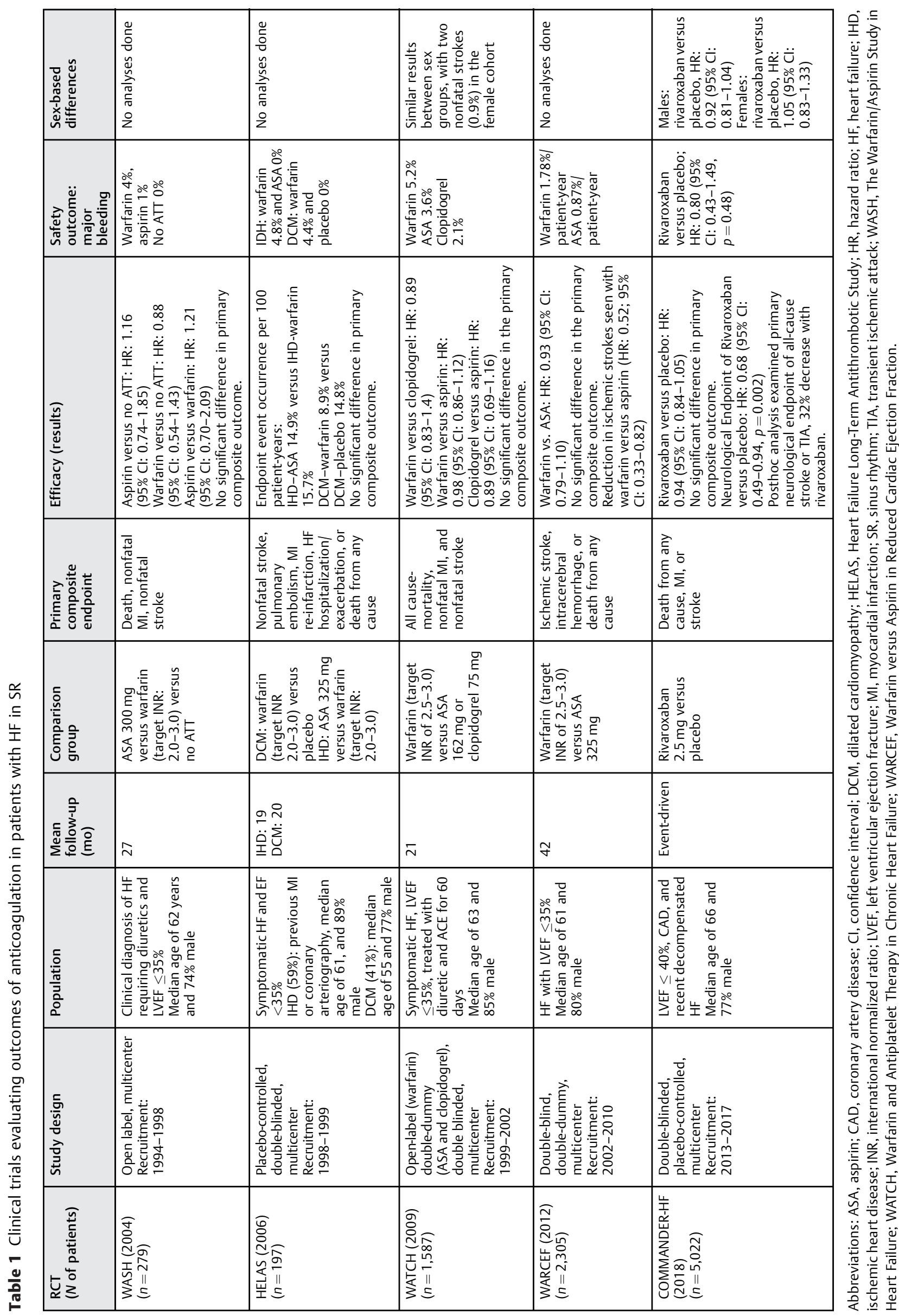




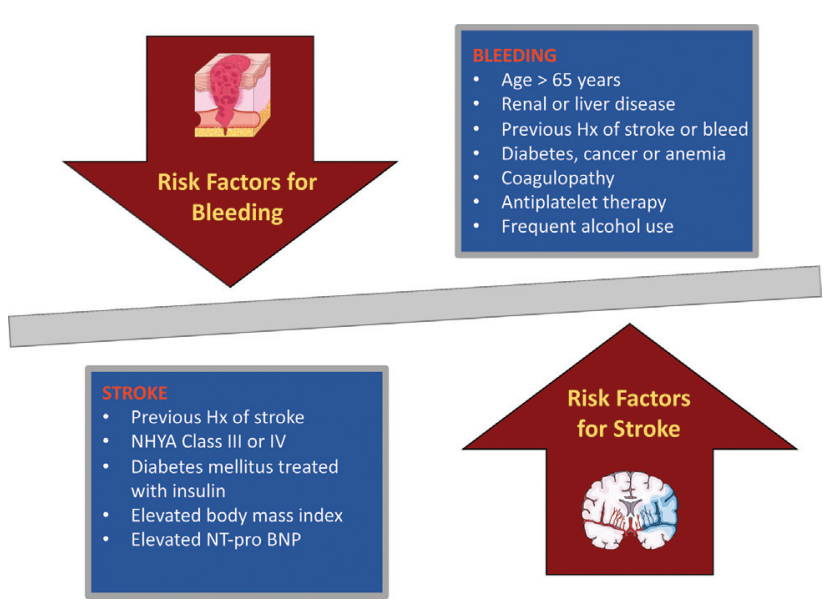

Fig. 3 Framework for balancing the risk factors for bleeding and for stroke in patients with heart failure in sinus rhythm.

which patients are at high risk for developing AF. A subanalysis of the COMMANDER-HF trial also suggested that including venous thromboembolism in the thromboembolic endpoints could better identify the subset of patients with HF and SR who may benefit from antithrombotic therapy. ${ }^{16}$

The $\mathrm{CHA}_{2} \mathrm{DS}_{2}$-VASc score (congestive HF, hypertension, age $\geq 75$ years, diabetes mellitus, prior stroke or thromboembolism, vascular disease, age 65 to 75 years, and female sex)-used to stratify the risk of stroke in patients with $\mathrm{AF}^{19,20}$-can predict thromboembolic events (ischemic stroke, transient ischemic attack, and peripheral arterial embolism) similarly well in patients in SR, ${ }^{21-23}$ as it reflects the abovementioned risk factors for thromboembolism in patients with $\mathrm{HF}$ and SR. Patients with AF and with two or more $\mathrm{CHA}_{2} \mathrm{DS}_{2}$-VASc risk factors have an almost threefold increased risk of stroke compared with those without any risk factors. ${ }^{24} \mathrm{~A}$ prospective cohort study which examined the predictive value of the $\mathrm{CHA}_{2} \mathrm{DS}_{2}$-VASc score in patients with $\mathrm{HF}$ in $\mathrm{SR}$ found a correlation between increasing scores and risk of ischemic stroke irrespective whether $\mathrm{AF}$ was present or not. ${ }^{20}$ Higher $\mathrm{CHA}_{2} \mathrm{DS}_{2}$-VASc scores $(\geq 4)$ in patients with $\mathrm{HF}$ were associated with a greater absolute risk of thromboembolism, irrespective of whether AF was present or not. The score demonstrated moderate discrimination for ischemic stroke but had a high negative predictive value for ischemic stroke and thromboembolism (92\%) at 1year follow-up for patients with $\mathrm{HF}$ and without $\mathrm{AF}^{20}$ The WARCEF trial also examined the accuracy of the $\mathrm{CHA}_{2} \mathrm{DS}_{2}-$ VASc in patients with HF in SR and found that it predicted adverse outcomes with moderate accuracy ${ }^{25}$ Despite modest predictive accuracy, these findings suggest that the $\mathrm{CHA}_{2} \mathrm{DS}_{2}$-VASc score could have clinical utility in detecting a subgroup of patients with HF who are at low risk as well as those at high risk of AF.

When determining which patients with AF would benefit from anticoagulation, an important consideration to balance continues to be the bleeding risk. A validated score used to identify patients at high bleeding risk is the HAS-BLED score (hypertension, abnormal renal/liver function, stroke, bleeding history or predisposition, labile international normalized ratio, age $>65$ years, and concomitant use of drugs/alcohol), which in conjunction with the $\mathrm{CHA}_{2} \mathrm{DS}_{2}$-VASc score can be helpful for clinical decision making when deciding whether to start anticoagulation therapy. ${ }^{26}$ However, there is evidence to suggest that increasing $\mathrm{CHA}_{2} \mathrm{DS}_{2}$-VASc scores correlate with an increased risk of bleeding, which is more than likely related to the overlap of shared risk factors for bleeding and stroke which are included in both risk scores. ${ }^{27}$ Predictors such as older age and previous stroke are associated with both an increased risk of bleeding and ischemic stroke. A recent study that looked at a cohort of over 4,000 patients with AF compared the predictive accuracy of the HAS-BLED score relative to the $\mathrm{CHA}_{2} \mathrm{DS}_{2}$-VASc score and revealed that in patients naïve to anticoagulation, the two scores had similar predictive values for major bleeding. ${ }^{27}$ In higher risk patients, the HAS-BLED score was found to have a slightly higher $c$-statistic compared with the $\mathrm{CHA}_{2} \mathrm{DS}_{2}$-VASc, but was still able to identify patients at high risk of bleeding. In patients with $\mathrm{HF}$ and $\mathrm{SR}$, the $\mathrm{CHA}_{2} \mathrm{DS}_{2}$-VASc score may therefore be useful in not only stratifying stroke risk but also bleeding risk. ${ }^{27}$ However, more evidence is needed to estimate the predictive accuracy and clinical utility of the $\mathrm{CHA}_{2} \mathrm{DS}_{2}$-VASc in a cohort that is specific to patients with $\mathrm{HF}$ in $\mathrm{SR}$.

Biomarkers may also have a role in identifying patients who may benefit from antithrombotic therapy; for example, D-dimer levels which may have a high predictive value for stroke risk. In the COMMANDER-HF trial, higher rates of stroke of any cause and ischemic stroke were associated with higher plasma D-dimer concentrations at baseline. ${ }^{22,28}$ More research regarding the mechanisms behind elevated D-dimer levels in patients with HF, their relevance, and whether screening for D-dimer levels can predict patients at risk of increased stroke is required.

Future studies could also explore the prognostic value of echocardiographic indicators including left ventricular (LV) wall hypertrophy, restrictive LV filling, and reduced global longitudinal strain (a measure of tissue deformation), which have all been associated with higher incidence of thromboembolic stroke in patients with $\mathrm{HF}^{21,22}$ These may be indicators of stroke risk but may also be on the causal pathway (-Table 2). The elevation in LV filling pressure is associated with left atrial remodeling and myopathy, which may be a risk factor for atrial dysrhythmias and thrombosis. $^{29}$

\section{Stroke: Rhythm and Risk Prediction}

Remote monitoring with intracardiac and wearable devices can detect atrial tachyarrhythmias and AF, both risk factors for stroke. ${ }^{23}$ The Asymptomatic Atrial Fibrillation and Stroke Evaluation in Pacemaker Patients and the Atrial Fibrillation Reduction Atrial Pacing Trial (ASSERT) assessed the burden of subclinical AF (incidence of stroke or thromboembolic events) in patients with intracardiac devices. While only $14 \%$ of patients in ASSERT had HF, the trial demonstrated that more than one-third of patients with implantable devices had subclinical atrial tachyarrhythmias (atrial rate $>190 \mathrm{bpm}$ for longer than 6 minutes), associated with a 
Table 2 Summary of knowledge gaps and areas for future research

\begin{tabular}{|l|l|}
\hline Important knowledge gaps & Areas of potential advancement \\
\hline $\begin{array}{l}\text { Detecting a subgroup of patients with HF and SR } \\
\text { who may benefit from anticoagulation }\end{array}$ & $\begin{array}{l}\text { A time-dependent anticoagulation approach where patients with HF and } \\
\text { transient AF are randomized to anticoagulation versus placebo following } \\
\text { the arrhythmia. }\end{array}$ \\
\hline $\begin{array}{l}\text { Determining clinical benefit of anticoagulation in } \\
\text { patients with HF in SR }\end{array}$ & $\begin{array}{l}\text { Selecting endpoints in clinical trials that are sensitive to antithrombotic } \\
\text { therapy such as thromboembolic events. }\end{array}$ \\
\hline Identifying risk of stroke in patients with HF in SR & $\begin{array}{l}\text { Integrating clinical variables, biomarkers, and echocardiographic } \\
\text { indicators. }\end{array}$ \\
\hline
\end{tabular}

Abbreviations: AF, atrial fibrillation; HF, heart failure; SR, sinus rhythm.

5.6-fold increased risk of AF and a 2.5-fold adjusted risk of stroke or systemic embolism. ${ }^{30}$ During the 3 months following device implantation, episodes of AF longer than 6 minutes in duration were associated with a 2.5 -fold increase in subsequent stroke. While these findings are not specific to HF, they add insight into factors that may be useful to risk stratify patients with HF.

A recent case-crossover study of 891 patients with ischemic stroke and intracardiac devices explored the temporal association between incidence of AF and risk of ischemic stroke. Heart rhythms 30 days prestroke (cases) were compared with those 91 to 120 days prestroke (controls) ${ }^{31} ; 52$ patients experienced AF episodes of 5.5 hours or more in the case period of 30 days in comparison to 14 patients in the control period-reflecting a 3.71-fold increased risk of stroke in weeks following multi-hour AF episodes. ${ }^{31}$ The risk of stroke was highest within the first 5 days after an AF episode. ${ }^{31}$ While this cohort was not specific to patients with HF and most patients did not meet the threshold of AF burden, these results suggest that remote monitoring for these variables could assist in risk stratification of patients (-Fig. 4).

Among patients with HF, remote monitoring has been shown to identify patients at high risk for stroke. ${ }^{32,33}$ Exploratory analysis of the remote management of HF using implantable electronic devices (REM-HF) trial of 1,650 patients with chronic HF revealed that $4.6 \%$ of patients had subclinical AF.

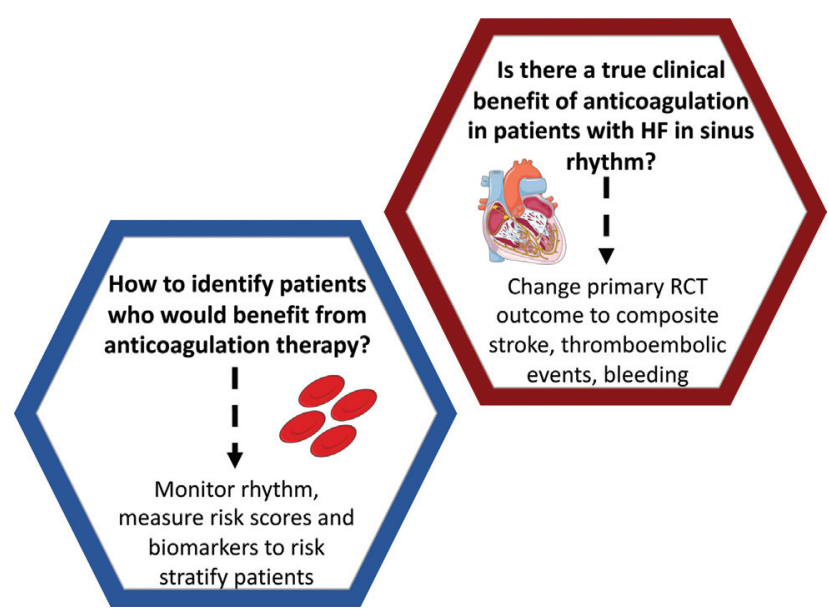

Fig. 4 Considerations in anticoagulation in patients with heart failure in sinus rhythm and future avenues to explore.
The incidence of ischemic stroke was highest in this subgroup, and AF was associated with increased cardiovascular hospitalization. $^{32}$ Mobile health and wearable technologies can expand remote monitoring to patients with HF who do not have intracardiac devices and have the potential to replace remote monitoring, although they will first require validation. ${ }^{34}$ The detection of subclinical atrial tachyarrhythmias and AF could identify a population of patients with HF at high risk of stroke who could be recruited into clinical trials of anticoagulation. Machine learning algorithms can also use electrocardiogram (ECG) patterns to identify patients at high risk for AF as well as stroke when standard ECG or Holter monitoring do not capture AF. Through identifying variables in both patients with and without AF as well as assessing ECG findings such as p-wave duration and cardiac biomarkers, models can be constructed to determine which variables can predict $\mathrm{AF}$ ( - Fig. 4). ${ }^{35,36}$

Given the association between atrial tachyarrhythmias, including transient $\mathrm{AF}$, and stroke as well as the proximity of stroke to AF episodes, a temporary time-dependent anticoagulation approach may be tested wherein patients with HF and these rhythms-as identified by wearable or intracardiac devices-are randomized to (temporary) anticoagulation versus placebo subsequent to the arrhythmia for a certain duration of time ( - Table 2 ). Such an approach may be the optimal way to decrease stroke burden while minimizing bleeding risk and merits a clinical trial.

\section{Conclusion}

Despite the high risk of stroke in patients with $\mathrm{HF}$, there is no clear evidence to support the use of antithrombotic therapy in those with SR and no other indications for anticoagulation. However, at least two of the five trials to date were inadequately powered and patients with HFpEF have not been included in any of the published trials. On balance, while anticoagulation decreased the risk of stroke, it did not decrease composite endpoints that include all-cause mortality, and its benefit is offset by an increased risk of major bleeding. However, most of the causes of death in patients with HF and SR are not amenable to risk reduction with anticoagulation. Thus, selection of endpoints that are sensitive to antithrombotic therapy may be an important consideration in future trials on the benefit of antithrombotic therapy. 
In addition to adequately powered trials with appropriate endpoints, determining which subgroups of patients are at particularly high risk of stroke through remote monitoring and clinical risk prediction models could guide selection of patients likely to derive benefit from antithrombotic therapy (-Fig. 4). Patients with HF and atrial tachyarrhythmias, including transient $\mathrm{AF}$, may represent a high-risk group that could be recruited into trials that test the efficacy of anticoagulation-possibly temporary and time-dependentgiven the proximity of stroke incidents to AF episodes. Wearable devices certainly afford the opportunity for a time-dependent anticoagulation approach, which may be the optimal way to decrease stroke burden while minimizing bleeding risk. Combining better patient selection and endpoint definitions in future trials will help to determine whether there is indeed a benefit of antithrombotic therapy in patients with HF and SR.

\section{Essentials}

- Patients with heart failure (HF) in sinus rhythm (SR) may have a high risk of thromboembolic events including stroke.

- Oral anticoagulation is well established in patients with HF and atrial fibrillation, but the benefit in SR remains uncertain as clinical trial results have been neutral and any reduction in stroke rates appears to be countered by bleeding events.

- Identifying patients who may benefit from anticoagulation within this group may require adequately powered trials that risk stratify patients with HF and SR prior to recruitment, and focus primarily on efficacy and safety outcomes that are sensitive to the intervention.

\section{Funding}

H.G.C.V.S. is funded by Canadian Institutes of Health Research and Heart and Stroke Foundation of Canada. R. B.S. has received funding from the European Research Council (ERC) under the European Union's Horizon 2020 research and innovation programme under the grant agreement No 648131, from the European Union's Horizon 2020 research and innovation programme under the grant agreement No 847770 (AFFECT-EU) and German Center for Cardiovascular Research (DZHK e.V.) (81Z1710103); German Ministry of Research and Education (BMBF 01ZX1408A) and ERACoSysMed3 (031L0239).

\section{Conflict of Interest}

None declared.

\section{References}

1 Lin AY, Dinatolo E, Metra M, et al. Thromboembolism in heart failure patients in sinus rhythm: epidemiology, pathophysiology, clinical trials, and future direction. JACC Hear Fail 2021;9(04): 243-253

2 Hjalmarsson C, Fu M, Zverkova Sandström T, et al. Risk of stroke in patients with heart failure and sinus rhythm: data from the Swedish Heart Failure Registry. ESC Hear Fail 2021;8(01):85-94
3 Zannad F, Stough WG, Regnault V, et al. Is thrombosis a contributor to heart failure pathophysiology? Possible mechanisms, therapeutic opportunities, and clinical investigation challenges. Int J Cardiol 2013;167(05):1772-1782

4 Abdul-Rahim AH, Perez A-C, Fulton RL, et al; Investigators of the Controlled Rosuvastatin Multinational Study in Heart Failure (CORONA) GISSI-Heart Failure (GISSI-HF) Committees and Investigators. Risk of stroke in chronic heart failure patients without atrial fibrillation: analysis of the Controlled Rosuvastatin in Multinational Trial Heart Failure (CORONA) and the Gruppo Italiano per lo Studio della Sopravvivenza nell'Insufficienza Cardiaca-Heart Failure (GISSI-HF) Trials. Circulation 2015;131(17): 1486-1494

5 Mehra MR, Vaduganathan M, Fu M, et al. A comprehensive analysis of the effects of rivaroxaban on stroke or transient ischaemic attack in patients with heart failure, coronary artery disease, and sinus rhythm: the COMMANDER HF trial. Eur Heart J 2019;40(44):3593-3602

6 Adelborg K, Szépligeti S, Sundbøll J, et al. Risk of stroke in patients with heart failure: a population-based 30-year cohort study. Stroke 2017;48(05):1161-1168

7 Scherbakov N, Haeusler KG, Doehner W. Ischemic stroke and heart failure: facts and numbers. ESC Hear Fail 2015;2(01):1-4

8 Kotecha D, Lam CSP, Van Veldhuisen DJ, Van Gelder IC, Voors AA, Rienstra M. Heart failure with preserved ejection fraction and atrial fibrillation: vicious twins. J Am Coll Cardiol 2016;68(20): 2217-2228

9 Gavaert AB, Kataria R, Zannad F, et al. Heart failure with preserved ejection fraction: recent concepts in diagnosis, mechanisms and management. Heart 2022. Doi: 10.1136/heartjnl-2021-319605

10 Hage C, Löfgren L, Michopoulos F, et al. Metabolomic profile in HFpEF vs HFrEF patients. J Card Fail 2020;26(12):1050-1059

$11 \mathrm{Uhm}$ J-S, Kim J, Yu HT, et al. Stroke and systemic embolism in patients with atrial fibrillation and heart failure according to heart failure type. ESC Heart Fail 2021;8(02):1582-1589

12 Zhang C, Wang N, Shen N-N, et al. Net clinical benefit of antithrombotic therapy in patients with heart failure and sinus rhythm: a network meta-analysis from 5 clinical trials. Thromb Res 2020; 190:122-128

13 Beggs SAS, Rørth R, Gardner RS, McMurray JJV. Anticoagulation therapy in heart failure and sinus rhythm: a systematic review and meta-analysis. Heart 2019;105(17):1325-1334

14 Sulaica EM, Macaulay TE, Helbing RR, Abo-Aly M, Abdel-Latif A, Wanat MA. A comparison of anticoagulation, antiplatelet, and placebo treatment for patients with heart failure reduced ejection fraction in sinus rhythm: a systematic review and meta-analysis. Heart Fail Rev 2020;25(02):207-216

15 Zannad F, Anker SD, Byra WM, et al; COMMANDER HF Investigators. Rivaroxaban in patients with heart failure, sinus rhythm, and coronary disease. N Engl J Med 2018;379(14):1332-1342

16 Greenberg B, Neaton JD, Anker SD, et al. Association of rivaroxaban with thromboembolic events in patients with heart failure, coronary disease, and sinus rhythm: a post hoc analysis of the COMMANDER HF trial. JAMA Cardiol 2019;4(06):515-523

17 Mujib M, Giamouzis G, Agha SA, et al. Epidemiology of stroke in chronic heart failure patients with normal sinus rhythm: findings from the DIG stroke sub-study. Int J Cardiol 2010;144(03): 389-393

18 Schnabel RB, Haeusler KG, Healey JS, et al. Searching for atrial fibrillation poststroke: a white paper of the AF-SCREEN international collaboration. Circulation 2019;140(22):1834-1850

$19 \mathrm{Kim} \mathrm{W,} \mathrm{Kim} \mathrm{EJ.} \mathrm{Heart} \mathrm{failure} \mathrm{as} \mathrm{a} \mathrm{risk} \mathrm{factor} \mathrm{for} \mathrm{stroke.} \mathrm{J} \mathrm{Stroke}$ 2018;20(01):33-45

20 Melgaard L, Gorst-Rasmussen A, Lane DA, Rasmussen LH, Larsen TB, Lip GYH. Assessment of the CHA2DS2-VASc score in predicting ischemic stroke, thromboembolism, and death in patients with heart failure with and without atrial fibrillation. JAMA 2015;314 (10):1030-1038 
21 Hijazi Z, Oldgren J, Lindbäck J, et al; ARISTOTLE and RE-LY Investigators. A biomarker-based risk score to predict death in patients with atrial fibrillation: the $\mathrm{ABC}$ (age, biomarkers, clinical history) death risk score. Eur Heart J 2018;39(06):477-485

22 Hijazi Z, Oldgren J, Siegbahn A, Wallentin L. Application of biomarkers for risk stratification in patients with atrial fibrillation. Clin Chem 2017;63(01):152-164

23 Siliste RN, Antohi EL, Pepoyan S, Nakou E, Vardas P. Anticoagulation in heart failure without atrial fibrillation: gaps and dilemmas in current clinical practice. Eur J Heart Fail 2018;20(06):978-988

24 Friberg L, Rosenqvist M, Lip GYH. Evaluation of risk stratification schemes for ischaemic stroke and bleeding in 182678 patients with atrial fibrillation: the Swedish Atrial Fibrillation cohort study. Eur Heart J 2012;33(12):1500-1510

25 Ye S, Qian M, Zhao B, et al; WARCEF Investigators. $\mathrm{CHA}_{2} \mathrm{DS}_{2}$-VASc score and adverse outcomes in patients with heart failure with reduced ejection fraction and sinus rhythm. Eur J Heart Fail 2016; 18(10):1261-1266

26 Pisters R, Lane DA, Nieuwlaat R, de Vos CB, Crijns HJGM, Lip GYH. A novel user-friendly score (HAS-BLED) to assess 1-year risk of major bleeding in patients with atrial fibrillation: the Euro Heart Survey. Chest 2010;138(05):1093-1100

27 Poli D, Antonucci E, Pengo V, Testa S, Palareti G. Comparison of HAS-BLED and HAS-BED versus $\mathrm{CHADS}_{2}$ and $\mathrm{CHA}_{2} \mathrm{DS}_{2}$ VASC stroke and bleeding scores in patients with atrial fibrillation. Am J Cardiol 2017;119(07):1012-1016

28 Ferreira JP, Lam CSP, Anker SD, et al. Plasma D-dimer concentrations predicting stroke risk and rivaroxaban benefit in patients with heart failure and sinus rhythm: an analysis from the COMMANDER-HF trial. Eur J Heart Fail 2021;23(04):648-656

29 Goette A, Kalman JM, Aguinaga L, et al. EHRA/HRS/APHRS/SOLAECE expert consensus on atrial cardiomyopathies: definition, characterisation, and clinical implication. J Arrhythm 2016;32(04):247-278

30 Healey JS, Connolly SJ, Gold MR, et al; ASSERT Investigators. Subclinical atrial fibrillation and the risk of stroke. N Engl J Med 2012;366(02):120-129

31 Singer DE, Ziegler PD, Koehler JL, Sarkar S, Passman RS. Temporal association between episodes of atrial fibrillation and risk of ischemic stroke. JAMA Cardiol 2021;6(12):1364-1369

32 Morgan JM, Kitt S, Gill J, et al. Remote management of heart failure using implantable electronic devices. Eur Heart J 2017;38(30): 2352-2360

33 Theuns DAMJ, Radhoe SP, Brugts JJ. Remote monitoring of heart failure in patients with implantable cardioverter-defibrillators: current status and future needs. Sensors (Basel) 2021;21(11): 3763

34 Lopez Perales CR, Van Spall HGC, Maeda S, et al. Mobile health applications for the detection of atrial fibrillation: a systematic review. EP Eur 2021;23(01):11-28

35 Kashou A, Noseworthy P. Predicting incident atrial fibrillation in sinus rhythm: more than just trusting the 'black box'.. Heart 2021; 107(22):1770-1771

36 Sanz-García A, Cecconi A, Vera A, et al. Electrocardiographic biomarkers to predict atrial fibrillation in sinus rhythm electrocardiograms. Heart (British Cardiac Society) 2021;107(22): 1813-1819 